\title{
A Star Image Extractor for the Nano-JASMINE satellite
}

\author{
M. Yamauchi ${ }^{12}$, N. Gouda ${ }^{1}$, Y. Kobayashi ${ }^{1}$, T. Tsujimoto ${ }^{1}$, T. Yano ${ }^{1}$, \\ M. Suganuma ${ }^{1}$, Y. Yamada ${ }^{3}$, S. Nakasuka ${ }^{2}$, N. Sako ${ }^{2}$ et al. \\ ${ }^{1}$ National Astronomical Observatory of Japan, \\ 2-21-1 Osawa, Mitaka, Tokyo, Japan \\ ${ }^{2}$ University of Tokyo, \\ 7-3-1 Hongo, Bunkyo-ku, Tokyo, Japan \\ ${ }^{3}$ Kyoto University, \\ Yoshida-Honmachi, Sakyo-ku, Kyoto, Japan \\ email: yamauchi@merope.mtk.nao.ac.jp
}

\begin{abstract}
We have developped a software of Star-Image-Extractor (SIE) which works as the onboard real-time image processor. It detects and extracts only the object data from raw image data. SIE has two functions: reducing image data and providing data for the satellite's high accuracy attitude control system.
\end{abstract}

Keywords. methods: data analysis, techniques: image processing

We have developed a software of Star Image Extractor (SIE) which works as the onboard real-time image processor. It detects and extracts only an object data from raw image data. SIE will be equipped to the Nano-JASMINE satellite. Nano-JASMINE is a small astrometry satellite that that observe objects in our galaxy. It will be launched in 2009 for a two year mission. Nano-JASMINE observes an object in the Time Delayed Integration (TDI) mode. TDI is one of the operation modes of CCD. The data are obtained, by reading out the CCD at a rate synchronized with a vertical charge transfer of CCD. The image data are sent thorough SIE to the Mission-controller. We show the data flow of the imaging system in Fig. 1

SIE has two purposes. One of the purposes is reducing image data. The original data rate of image is $2 \mathrm{Mb}$ per second. The amount of raw images is too large to be transmitted to the ground, so we use only extracted images for the transmission. SIE reduces the data rate from $2 \mathrm{Mbps}$ to $\mathrm{N} \times 50 \mathrm{bps}$, where $\mathrm{N}$ is the averaged number of detected objects in one field of view. Nano-JASMINE should be able to detect a few stars each second. Another purpose is to make the data available for the satellite's high accuracy attitude control system. The extracted image is used as a rotation sensor, because the point-spread function of an object will be elongated more in the TDI direction.

A software simulator of SIE has been developed to test the star extracting algorithm. FITS formatted files are used for input and output data of the simulation. Two kinds of input data are used for the simulation. One is the image that obtained by the imaging experiments using the hardware of Nano-JASMINE imaging system. Another is the image, produced by the software simulator, which has no background noise. The simulation experiments have shown that we can extract stars from the input images successfully. Results of our simulations are shown in Fig. 2. The software simulator has been finished. Now the logic circuit is under development. The logic circuit will be written on a Field Programmable Gate Array (FPGA) device. 


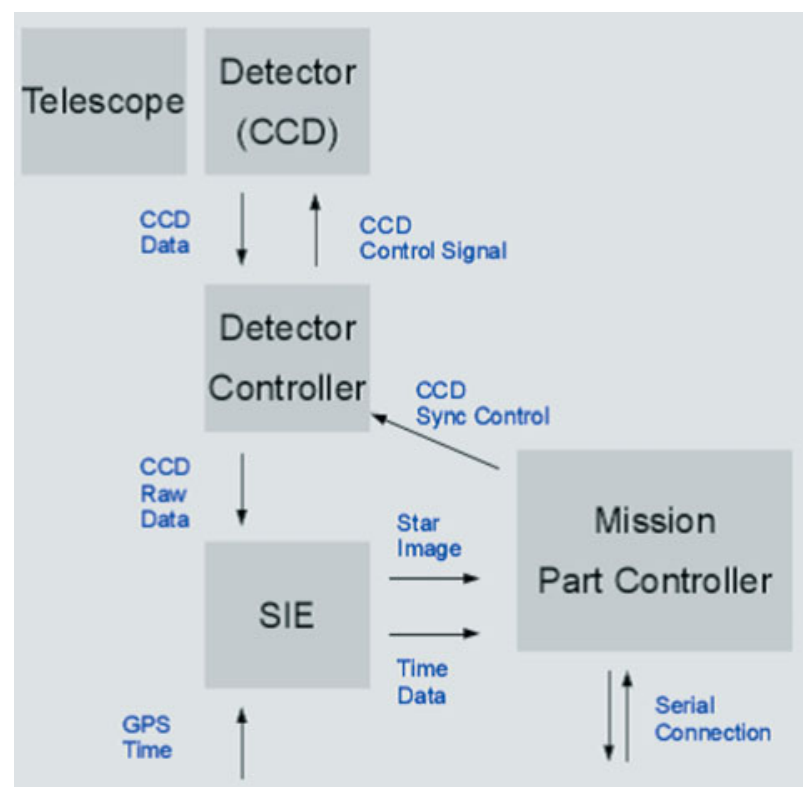

Figure 1. Data flow of imaging system

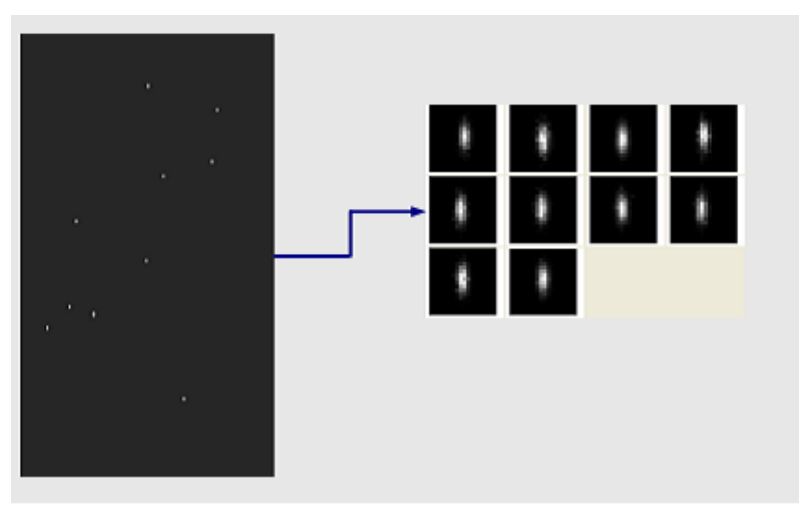

Figure 2. Simulation result (Extracting stars)

\section{References}

Hirte, S. \& Scholz, R. 2002, Die Verarbeitung des Rohdatenflusses eines Weltrauminterferometers, Schlussbericht zur Studie, Astrophysikalisches Institut Potsdam

Kovalevsky, J. 2001, Modern Astrometry(Second Edition), Springer

Takagi, M. \& Shimoda, H. 2004, Handbook of Image Analysis, University of Tokyo Press 